\title{
Acute toxicity of the cyfluthrin pesticide on guppy fish
}

Mahmut Selvi · Rabia Sarkaya •

Figen Erkoç • Oner Koçak

Received: 15 September 2007/ Accepted: 5 February 2008

(C) Springer-Verlag 2008

This article has been retracted due to copyright issues that cannot be resolved.

M. Selvi (凹) · R. Sarıkaya · F. Erkoç · O. Koçak

Department of Biology Education, Gazi University,

Teknikokullar, 06500 Ankara, Turkey

e-mail: mselvi@gazi.edu.tr 